Working Paper Series

ISSN 1170-487X

\title{
Benchmarking Attribute Selection Techniques for Discrete Class Data Mining
}

\section{Mark Hall and Geoffrey Holmes}

Working Paper: 02/02

April 2002

\author{
(C) 2002 Mark Hall and Geoffrey Holmes \\ Department of Computer Science \\ The University of Waikato \\ Private Bag 3105 \\ Hamilton, New Zealand
}




\title{
Benchmarking Attribute Selection Techniques for Discrete Class Data Mining
}

\author{
Mark A. Hall, Geoffrey Holmes
}

April 18, 2002

\begin{abstract}
Data engineering is generally considered to be a central issue in the development of data mining applications. The success of many learning schemes, in their attempts to construct models of data, hinges on the reliable identification of a small set of highly predictive attributes. The inclusion of irrelevant, redundant and noisy attributes in the model building process phase can result in poor predictive performance and increased computation.

Attribute selection generally involves a combination of search and attribute utility estimation plus evaluation with respect to specific learning schemes. This leads to a large number of possible permutations and has led to a situation where very few benchmark studies have been conducted.

This paper presents a benchmark comparison of several attribute selection methods for supervised classification. All the methods produce an attribute ranking, a useful devise for isolating the individual merit of an attribute. Attribute selection is achieved by cross-validating the attribute rankings with respect to a classification learner to find the best attributes. Results are reported for a selection of standard data sets and two diverse learning schemes $\mathrm{C} 4.5$ and naive Bayes.
\end{abstract}

\section{Introduction}

Many factors affect the success of data mining algorithms on a given task. The quality of the data is one such factor--if information is irrelevant or redundant, or the data is noisy and unreliable, then knowledge discovery during training is more difficult. Attribute subset selection is the process of identifying and removing as much of the irrelevant and redundant information as possible. Learning algorithms differ in the amount of emphasis they place on attribute selection. At one extreme are algorithms such as the simple nearest neighbour learner, that classifies novel examples by retrieving the nearest stored training example, using all the available features in its distance computations. At the other extreme are algorithms that explicitly try to focus on relevant features and ignore irrelevant ones. Decision tree inducers are examples of this approach. By testing the values of certain attributes, decision tree algorithms attempt to divide training 
data into subsets containing a strong majority of one class. This necessitates the selection of a small number of highly predictive features in order to avoid over fitting the training data. Regardless of whether a leamer attempts to select attributes itself or ignores the issue, attribute selection prior to learning can be beneficial. Reducing the dimensionality of the data reduces the size of the hypothesis space and allows algorithms to operate faster and more effectively. In some cases accuracy on future classification can be improved; in others, the result is a more compact, easily interpreted representation of the target concept.

Many attribute selection methods approach the task as a search problem, where each state in the search space specifies a distinct subset of the possible attributes [3]. Since the space is exponential in the number of attributes, this necessitates the use of a heuristic search procedure for all but trivial data sets. The search procedure is combined with an attribute utility estimator in order to evaluate the relative merit of alternative subsets of attributes. When the evaluation of the selected features with respect to learning algorithms is considered as well it leads to a large number of possible permutations. This fact, along with the computational cost of some attribute selection techniques, has led to a situation where very few benchmark studies on non-trivial data sets have been conducted.

Good surveys reviewing work in machine learning on feature selection can be found in $[3,4]$. In particular, Liu et. al. [4] use small artificial data sets to explore the strengths and weaknesses of different attribute selection methods with respect to issues such as noise, different attribute types, multi-class data sets and computational complexity. This paper, on the other hand, provides an empirical comparison of six major attribute selection methods on fourteen well known benchmark data sets for classification. Performance on a further three "large" data sets (two containing several hundreds of features, and the third over a thousand features) is reported as well. In this paper we focus on attribute selection techniques that produce ranked lists of attributes. These methods are not only useful for improving the performance of learning algorithms; the rankings they produce can also provide the data miner with insight into their data by clearly demonstrating the relative merit of individual attributes. The next section describes the attribute selection techniques compared in the benchmark. Section 3 outlines the experimental methodology used and briefly describes the Weka Experiment Editor (a powerful Java based system that was used to run the benchmarking experiments). Sections 4 and 5 present the results. The last section summarises the findings.

\section{Attribute Selection Techniques}

Attribute selection techniques can be categorised according to a number of criteria. One popular categorisation has coined the terms "filter" and "wrapper" to describe the nature of the metric used to evaluate the worth of attributes [10]. Wrappers evaluate attributes by using accuracy estimates provided by the actual target learning algorithm. Filters, on the other hand, use general 
characteristics of the data to evaluate attributes and operate independently of any learning algorithm. Another useful taxonomy can be drawn by dividing algorithms into those which evaluate (and hence rank) individual attributes and those which evaluate (and hence rank) subsets of attributes. The latter group can be split further on the basis of the search technique commonly employed with each method to explore the space of attribute subsets ${ }^{1}$. Some attribute selection techniques can handle regression problems, that is, when the class is a numeric rather than a discrete valued variable. This provides yet another dimension to categorise methods. Although some of the methods compared herein are capable of handling regression problems, this study has been restricted to discrete class data sets as all the methods are capable of handling this sort of problem.

By focusing on techniques that rank attributes we have simplified the matter by reducing the number of possible permutations. That is not to say that we have ignored those methods that evaluate subsets of attributes; on the contrary, it is possible to obtain ranked lists of attributes from these methods by using a simple hill climbing search and forcing it to continue to the far side of the search space. For example, forward selection hill climbing search starts with an empty set and evaluates each attribute individually to find the best single attribute. It then tries each of the remaining attributes in conjunction with the best to find the best pair of attributes. This process continues until no single attribute addition improves the evaluation of the subset. By forcing the search to continue (even though the best attribute added at each step may actually decrease the evaluation of the subset as a whole) and by noting each attribute as it is added, a list of attributes ranked according to their incremental improvement to the subset is obtained. Figure 1 demonstrates this process graphically.

Several of the attribute selection techniques compared in the benchmark only operate on discrete valued features. In order to apply these techniques to data with numeric features discretisation is applied as a preprocessing step. We used the state-of-the-art supervised discretisation technique developed by Fayyad and Irani [6]. Essentially the technique combines an entropy based splitting criterion (such as that used by the C4.5 decision tree learner [14]) with a minimum description length stopping criterion. The best cut point is the one that makes the subintervals as pure as possible, i.e where the information value is smallest (this is the same as splitting where the information gain, defined as the difference between the information value without the split and that with the split, is largest). The method is then applied recursively to the two subintervals until it is time to stop. For a set of instances $S$, a feature $A$ and a cut point $T$, the class information entropy of the partition created by $T$ is given by

$$
E(A ; T ; S)=\frac{\left|S_{1}\right|}{|S|} \operatorname{Ent}\left(S_{1}\right)+\frac{\left|S_{2}\right|}{|S|} \operatorname{Ent} S_{2}
$$

\footnotetext{
${ }^{1}$ It is important to note that any search technique can be used with a method that evaluates attribute subsets and that many of the possible permutations that this leads to have yet to be explored
} 


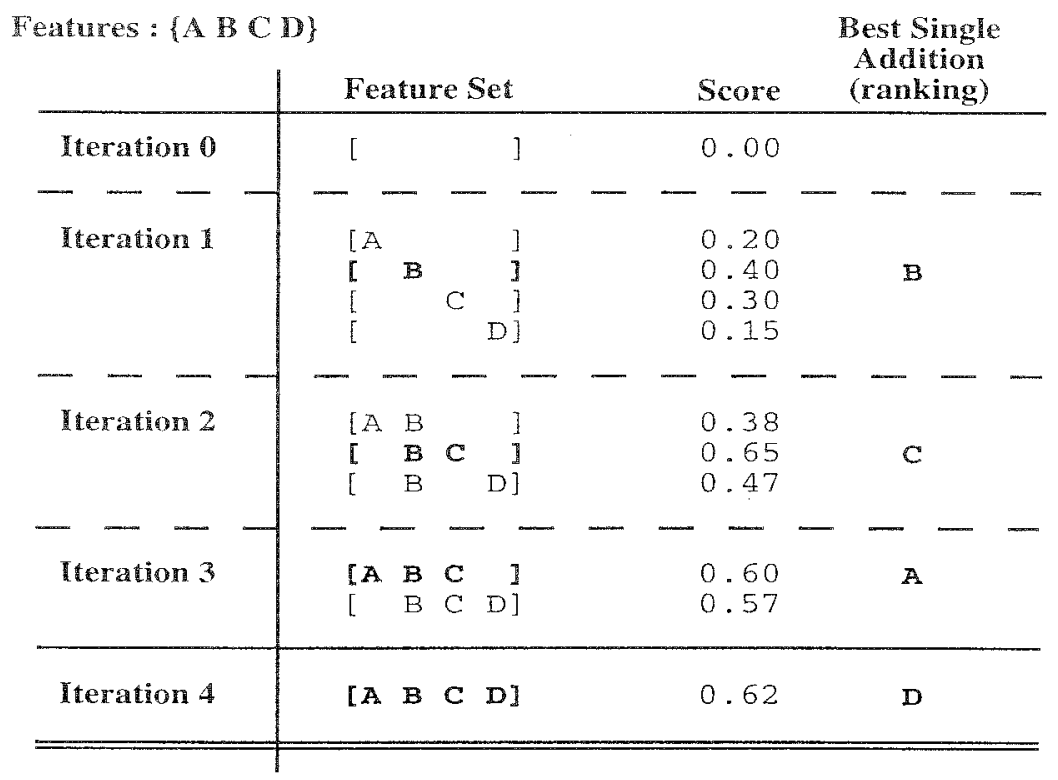

Figure 1: Forward selection search modified to produce a ranked list of attributes. Normally the search would terminate after iteration 3 because no single attribute addition improves the best subset from iteration 2 . In this case the search has been forced to continue until all attributes have been included. 
where $S_{1}$ and $S_{2}$ are two intervals of $S$ bounded by cut point $T$, and $\operatorname{Ent}(S)$ is the class entropy of a subset $S$ given by

$$
\operatorname{Ent}(S)=\sum_{i=1}^{C} p\left(C_{i}, S\right) \log _{2}\left(p\left(C_{i}, S\right)\right)
$$

The stopping criterion prescribes accepting a partition $T$ if and only if the cost of encoding the partition and the classes of the instances in the intervals induced by $T$ is less than the cost of encoding the classes of the instances before splitting. The partition created by $T$ is accepted iff

$\operatorname{Gain}(A ; T ; S)>\frac{\log _{2}(N-1)}{N}+\frac{\log _{2}\left(3^{c}-2\right)-\left[c \operatorname{Ent}(S)-c_{1} \operatorname{Ent}\left(S_{1}\right)-c_{2} \operatorname{Ent}\left(S_{2}\right)\right]}{N}$

In Equation 3, N is the number of instances, $c, c_{1}$, and $c_{2}$ are the number of distinct classes present in $S, S_{1}$, and $S_{2}$ respectively. The first component is the information needed to specify the splitting point; the second is a correction due to the need to transmit which classes correspond to the upper and lower subintervals.

The rest of this section is devoted to a brief description of each of the attribute selection methods compared in the benchmark. There are three methods that evaluate individual attributes and produce a ranking unassisted, and a further three methods which evaluate subsets of attributes. The forward selection search method described above is used with these last three methods to produce ranked lists of attributes. The methods cover major developments in attribute selection for machine learning over the last decade. We also include a classical statistical technique for dimensionality reduction.

\subsection{Information Gain Attribute Ranking}

This is one of the simplest (and fastest) attribute ranking methods and is often used in text categorisation applications $[5,16]$ where the sheer dimensionality of the data precludes more sophisticated attribute selection techniques. If $A$ is an attribute and $C$ is the class, Equations 4 and 5 give the entropy of the class before and after observing the attribute.

$$
\begin{gathered}
H(C)=-\sum_{c \in C} p(c) \log _{2} p(c) \\
H(C \mid A)=-\sum_{a \in A} p(a) \sum_{c \in C} p(c \mid a) \log _{2} p(c \mid a) .
\end{gathered}
$$

The amount by which the entropy of the class decreases reflects the additional information about the class provided by the attribute and is called information gain [14].

Each attribute $A_{i}$ is assigned a score based on the information gain between itself and the class: 


$$
\begin{aligned}
\mathrm{IG}_{i} & =H(C)-H\left(C \mid A_{i}\right) \\
& =H\left(A_{i}\right)-H\left(A_{i} \mid C\right) \\
& =H\left(A_{i}\right)+H(C)-H\left(A_{i}, C\right) .
\end{aligned}
$$

Data sets with numeric attributes are first discretized using the method of Fayyad and Irani [6].

\section{2 - Relief}

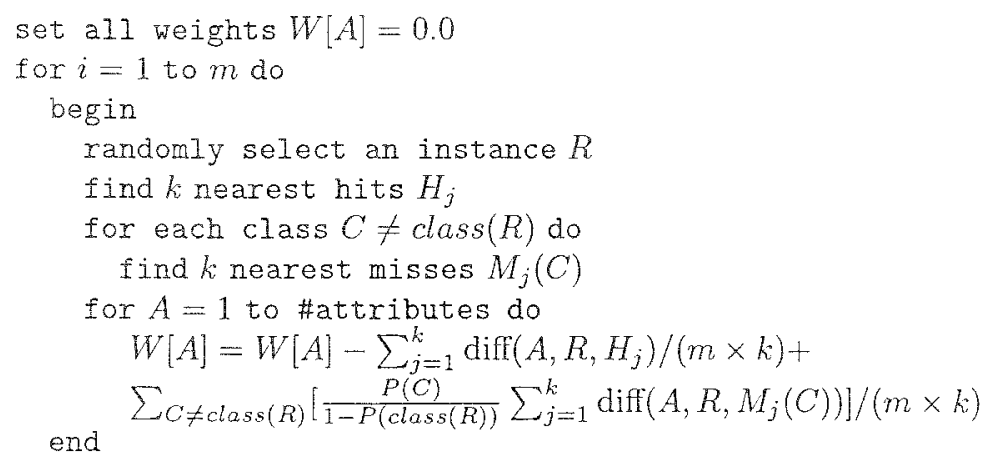

Figure 2: ReliefF algorithm.

Relief is an instance based attribute ranking scheme introduced by Kira and Rendell [9] and later enhanced by Kononenko [11]. Relief works by randomly sampling an instance from the data and then locating its nearest neighbour from the same and opposite class. The values of the attributes of the nearest neighbours are compared to the sampled instance and used to update relevance scores for each attribute. This process is repeated for a user specified number of instances $m$. The rationale is that a useful attribute should differentiate between instances from different classes and have the same value for instances from the same class.

Relief was originally defined for two-class problems and was later extended (ReliefF) to handle noise and multi-class data sets [11]. ReliefF smoothes the influence of noise in the data by averaging the contribution of $k$ nearest neighbours from the same and opposite class of each sampled instance instead of the single nearest neighbour. Multi-class data sets are handled by finding nearest neighbours from each class that is different from the current sampled instance and weighting their contributions by the prior probability of each class. Figure 2 shows the ReliefF algorithm. The function $\operatorname{diff}$ (Attribute, Instance 1, Instance 2 ) computes the difference between the values of Attribute for two instances. For discrete attributes the difference is either 
1 (the values are different) or 0 (the values are the same), while for continuous attributes the difference is the actual difference normalised to the interval $[0,1]$.

Kononenko [11] notes that the higher the value of $m$ (the number of instances sampled), the more reliable ReliefF's estimates are-though of course increasing $m$ increases the running time. For all experiments reported in this paper, we set $m=250$ and $k=10[11,15]$.

\subsection{Principal Components}

Principal component analysis is a statistical technique that can reduce the dimensionality of data as a by-product of transforming the original attribute space. Transformed attributes are formed by first computing the covariance matrix of the original attributes, and then extracting its eigenvectors. The eigenvectors (principal components) define a linear transformation from the original attribute space to a new space in which attributes are uncorrelated. Eigenvectors can be ranked according to the amount of variation in the original data that they account for. Typically the first few transformed attributes account for most of the variation in the data and are retained, while the remainder are discarded.

It is worth noting that of all the attribute selection techniques compared, principal components is the only unsupervised method - that is, it makes no use of the class attribute. Our implementation of principal components handles $k$-valued discrete attributes by converting them to $k$ binary attributes. Each of these attributes has a ' 1 ' for every occurrence of the corresponding $k$ 'th value of the discrete attribute, and a ' 0 ' for all other values. These new synthetic binary attributes are then treated as numeric attributes in the normal manner. This has the disadvantage of increasing the dimensionality of the original space when multi-valued discrete attributes are present.

\subsection{CFS}

CFS (Correlation-based Feature Selection) $[7,8]$ is the first of the methods that evaluate subsets of attributes rather than individual attributes. At the heart of the algorithm is a subset evaluation heuristic that takes into account the usefulness of individual features for predicting the class along with the level of inter-correlation among them. The heuristic (Equation 7) assigns high scores to subsets containing attributes that are highly correlated with the class and have low inter-correlation with each other.

$$
\text { Merit }_{s}=\frac{k \overline{r_{c f}}}{\sqrt{k+k(k-1) \overline{r_{f f}}}},
$$

where Merit $S$ is the heuristic "merit" of a feature subset $S$ containing $k$ features, $\overline{r_{c f}}$ the average feature-class correlation, and $\overline{r_{f f}}$ the average feature-feature inter-correlation. The numerator can be thought of as giving an indication of how predictive a group of features are; the denominator of how much redundancy there is among them. The heuristic handles irrelevant features as they will be poor predictors of the class. Redundant attributes are discriminated against as 
they will be highly correlated with one or more of the other features. Because attributes are treated independently, CFS cannot identify strongly interacting features such as in a parity problem. However, it has been shown that it can identify useful attributes under moderate levels of interaction [7].

In order to apply Equation 7 it is necessary to compute the correlation (dependence) between attributes. CFS first discretizes numeric features using the technique of Fayyad and Irani [6] and then uses symmetrical uncertainty to estimate the degree of association between discrete features $(X$ and $Y$ ):

$$
S U=2.0 \times\left[\frac{H(X)+H(Y)-H(X, Y)}{H(X)+H(Y)}\right] .
$$

After computing a correlation matrix CFS applies a heuristic search strategy to find a good subset of features according to Equation 7. As mentioned at the start of this section we use the modified forward selection search, which produces a list of attributes ranked according to their contribution to the goodness of the set.

\subsection{Consistency-based Subset Evaluation}

Several approaches to attribute subset selection use class consistency as an evaluation metric $[1,13]$. These methods look for combinations of attributes whose values divide the data into subsets containing a strong single class majority. Usually the search is biased in favour of small feature subsets with high class consistency. Our consistency-based subset evaluator uses Liu and Setiono's [13] consistency metric:

$$
\text { Consistency }=1-\frac{\sum_{i=0}^{J}\left|D_{i}\right|-\left|M_{i}\right|}{N}
$$

where $s$ is an attribute subset, $J$ is the number of distinct combinations of attribute values for $s,\left|D_{i}\right|$ is the number of occurrences of the $i$ th attribute value combination, $\left|M_{i}\right|$ is the cardinality of the majority class for the $i$ th attribute value combination and $N$ is the total number of instances in the data set.

Data sets with numeric attributes are first discretized using the method of Fayyad and Irani [6]. The modified forward selection search described at the start of this section is used to produced a list of attributes, ranked according to their overall contribution to the consistency of the attribute set.

\subsection{Wrapper Subset Evaluation}

As described at the start of this section Wrapper attribute selection uses a target learning algorithm to estimate the worth of attribute subsets. Cross-validation is used to provide an estimate for the accuracy of a classifier on novel data when using only the attributes in a given subset. Our implementation uses repeated five-fold cross-validation for accuracy estimation. Cross-validation is repeated as long as the standard deviation over the runs is greater than one percent of the mean accuracy or until five repetitions have been completed [10]. The 
modified forward selection search described at the start of this section is used to produced a list of attributes, ranked according to their overall contribution to the accuracy of the attribute set with respect to the target learning algorithm.

Wrappers generally give better results than filters because of the interaction between the search and the learning scheme's inductive bias. But improved performance comes at the cost of computational expense-a result of having to invoke the learning algorithm for every attribute subset considered during the search.

\section{Experimental Methodology}

Our benchmark experiment applied the six attribute selection techniques to sixteen standard machine learning data sets from the UCI collection [2]. These data sets range in size from less than 100 instances up to several thousand, with each having less than 100 attributes. A further three data sets (also available from the UCI repository) are included in order to see how the attribute selection techniques fare in situations where there are larger numbers of features. The full characteristics of all the data sets are summarised in Table 1. In order to compare the effectiveness of attribute selection, attribute sets chosen by each technique were tested with two learning algorithms-a decision tree learner (C4.5 release 8) and a probabilistic learner (naive Bayes). These two algorithms were chosen because they represent two quite different approaches to learning and they are relatively fast, state-of-the-art algorithms that are often used in data mining applications. The naive Bayes algorithm employs a simplified version of Bayes formula to decide which class a test instance belongs to. The posterior probability of each class is calculated, given the feature values present in the instance; the instance is assigned to the class with the highest probability. Naive Bayes makes the assumption that feature values are statistically independent given the class. Learning a naive Bayes classifier is straightforward and involves simply estimating the probability of attribute values within each class from the training instances. Simple frequency counts are used to estimate the probability of discrete attribute values. For numeric attributes it is common practise to use the normal distribution [12]. C4.5 is an algorithm that summarises the training data in the form of a decision tree. Learning a decision tree is a fundamentally different process than learning a naive Bayes model. C4.5 recursively partitions the training data according to tests on attribute values in order to separate the classes. Although attribute tests are chosen one at a time in a greedy manner, they are dependent on results of previous tests.

For the first sixteen data sets in Table 1 the percentage of correct classifications, averaged over ten ten-fold cross validation runs, were calculated for each algorithm-data set combination before and after attribute selection. For each train-test split, the dimensionality was reduced by each attribute selector before being passed to the learning algorithms. Dimensionality reduction was accomplished by cross validating the attribute rankings produced by each attribute selector with respect to the current learning algorithm. That is, ten-fold cross 
Table 1: Data sets.

\begin{tabular}{llllll}
\hline Data Set & Train size & Test size & Num. & Nom. & Classes \\
\hline glass-2 & 163 & CV & 9 & 0 & 2 \\
anneal & 898 & CV & 6 & 32 & 5 \\
breast-c & 286 & CV & 0 & 9 & 2 \\
credit-g & 1000 & CV & 7 & 13 & 2 \\
diabetes & 768 & CV & 8 & 0 & 2 \\
horse colic & 368 & CV & 7 & 15 & 2 \\
heart-c & 303 & CV & 6 & 7 & 2 \\
heart-stat & 270 & CV & 13 & 0 & 2 \\
ionosphere & 351 & CV & 34 & 0 & 2 \\
labor & 57 & CV & 8 & 8 & 2 \\
lymph & 148 & CV & 3 & 15 & 4 \\
segrnent & 2310 & CV & 19 & 0 & 7 \\
soybean & 683 & CV & 0 & 35 & 19 \\
vote & 435 & CV & 0 & 16 & 2 \\
zoo & 101 & CV & 1 & 16 & 7 \\
\hline arrhythmia & 298 & 154 & 206 & 73 & 13 \\
anonymous & 29589 & 11122 & 0 & 293 & 2 \\
internet-ads & 2164 & 115 & 2 & 1555 & 2 \\
\hline
\end{tabular}

validation on the training part of each train-test split was used to estimate the worth of the highest ranked attribute, the first two highest ranked attributes, the first three highest ranked attributes etc. The highest $n$ ranked attributes with the best cross validated accuracy was chosen as the best subset. The last three data sets in Table 1 were split into a training set containing two thirds of the data and a test set containing the remaining data. Attribute selection was performed using the training data and each learning scheme was tested using the selected features on the test data.

For the attribute selection techniques that require data pre-processing, a copy of each training split was made for them to operate on. It is important to note that pre-processed data was only used during the attribute selection process, and with the exception of Principal components-where data transformation occurs-original data (albeit dimensionally reduced) was passed to each learning scheme. The same folds were used for each attribute selector-learning scheme combination. Although final accuracy of the induced models using the reduced feature sets was of primary interest, we also recorded statistics such as the number of attributes selected, time taken to select attributes and the size of the decision trees induced by $\mathrm{C} 4.5$.

\subsection{Weka Experiment Editor}

To perform the benchmark experiment we used $\mathrm{Weka}^{2}$ (Waikato Environment for Knowledge Analysis) - a powerful open-source Java-based machine learning workbench that can be run on any computer that has a Java run time environment installed. Weka brings together many machine learning algorithms and

\footnotetext{
${ }^{2}$ Weka is freely available from http://www.cs.waikato.ac.nz/ $\sim \mathrm{ml}$
} 


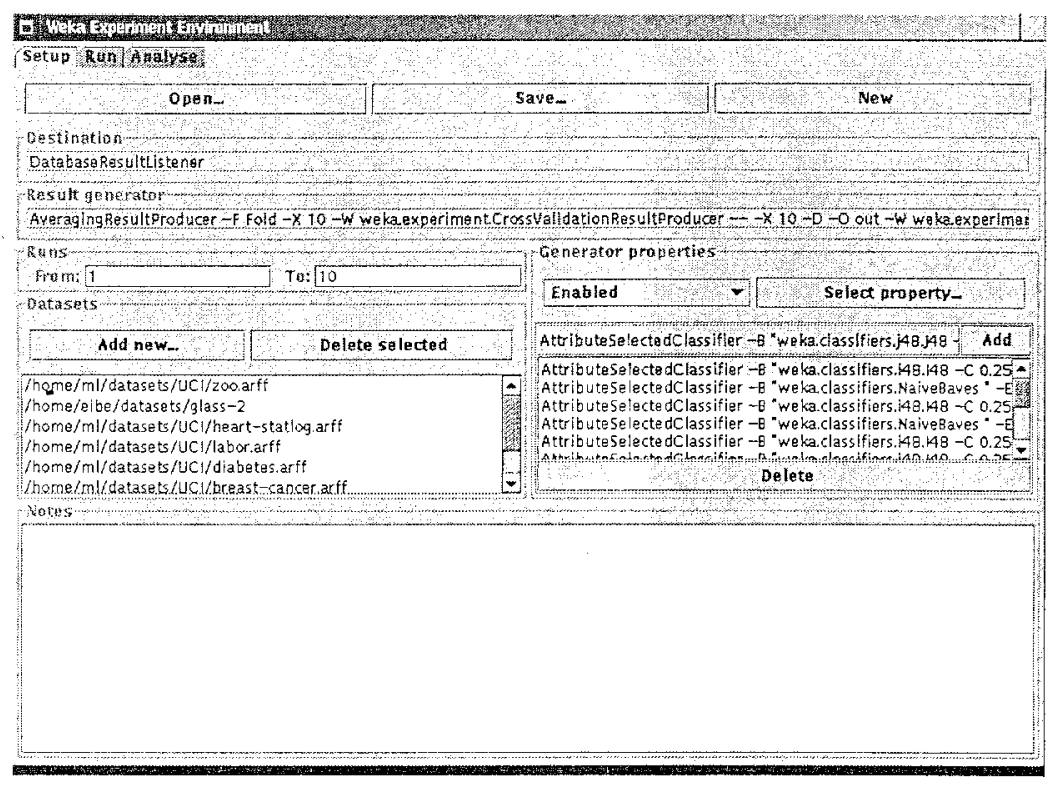

Figure 3: Weka Experimenter.

tools under a common framework with an intuitive graphical user interface. Weka has two primary modes: a data exploration mode and an experiment mode. The data exploration mode (Explorer) provides easy access to all of Weka's data preprocessing, learning, attribute selection and data visualisation modules in an environment that encourages initial exploration of the data. The experiment mode (Experimenter) allows large scale experiments to be run with results stored in a database for later retrieval and analysis. Figure 3 shows the configuration panel of the Experimenter.

\section{Results on Sixteen Benchmark Data Sets}

Table 2 shows the results on the first sixteen data sets for attribute selection with naive Bayes. The table shows how often each method performs significantly better (denoted by a o) or worse (denoted by $\mathbf{\bullet} \bullet$ ) than performing no feature selection (column 2). Throughout we speak of results being significantly different if the difference is statistically significant at the $1 \%$ level according to a paired two-sided $t$ test. From Table 2 it can be seen that the best result is from the Wrapper which improves naive Bayes on six data sets and degrades it on two. CFS is second best with improvement on five datasets and degradation on three. The simple information gain technique (IG) results in six improvements and four degradations. The consistency method (CNS) improves naive Bayes on six data sets and degrades it on five. ReliefF gives better performance on seven 
Table 2: Results for attribute selection with naive Bayes

\begin{tabular}{lllllllll}
\hline Data Set & NB & IG & RLF & CNS & PC & CFS & WRP \\
\hline zoo & 95.04 & 94.34 & 93.37 & 93.85 & 93.86 & 93.94 & 94.34 \\
heart-c & 83.83 & $82.54 *$ & 82.12 & 82.28 & 81.85 & 82.64 & 82.68 \\
ionosphere & 82.6 & $88.78 \circ$ & $89.52 \circ$ & $89.95 \circ$ & $90.72 \circ$ & $89.75 \circ$ & $91.28 \circ$ \\
soybean & 92.9 & $92.43 \bullet$ & $92.56 \bullet$ & 92.81 & 90.93 & 92.46 & 92.64. \\
glass2 & 62.33 & $67.42 \circ$ & $63.83 \circ$ & $68.31 \circ$ & $66.74 \circ$ & $71.08 \circ$ & $75.06 \circ$ \\
vote & 90.19 & $95.63 \circ$ & $95.33 \circ$ & $95.82 \circ$ & $92.32 \circ$ & $95.63 \circ$ & $95.93 \circ$ \\
heart-stat & 84.37 & 85.11 & 86 & $\circ$ & 83.48 & $82.07 \bullet$ & 85.07 & 85 \\
lymph & 83.24 & 82.63 & $81.47 \bullet$ & 82.55 & $79.67 \bullet$ & 82.35 & 84.11 \\
labor & 93.93 & $89.17 \bullet$ & $90.97 \bullet$ & 92 & $89.77 \bullet$ & 89.2 & 85.77 \\
diabetes & 75.73 & 76.24 & 75.95 & 75.64 & $74.42 \bullet$ & 76.19 & 76.12 \\
breast-c & 73.12 & 72.84 & $70.99 \bullet$ & 71.79 & 73.54 & 73.01 & 72.28 \\
credit-g & 74.98 & 74.36 & $74.49 \bullet$ & $74.06 \bullet$ & $73.3 \bullet$ & 74.33 & 74.35 \\
segment & 80.1 & $87.17 \circ$ & $86.97 \circ$ & $85.98 \circ$ & $90.03 \circ$ & $89.03 \circ$ & $89.57 \circ$ \\
horse colic & 78.28 & $83.2 \circ$ & $82.58 \circ$ & $82.77 \circ$ & 78.56 & $83.01 \circ$ & $82.61 \circ$ \\
anneal & 86.51 & $87.06 \circ$ & $89.17 \circ$ & $89.71 \circ$ & $90.65 \circ$ & 87.16 & $92.91 \circ$ \\
\hline
\end{tabular}

Table 3: Wins versus losses for accuracy of attribute selection with naive Bayes.

\begin{tabular}{rlll}
\hline Scheme & $\begin{array}{l}\text { Wins- } \\
\text { Losses }\end{array}$ & Wins & Losses \\
\hline WRP & 30 & 34 & 4 \\
CFS & 7 & 21 & 14 \\
CNS & 2 & 21 & 19 \\
IG & -2 & 17 & 19 \\
RLF & -3 & 19 & 22 \\
NB & -7 & 28 & 35 \\
PC & -27 & 17 & 44 \\
\hline
\end{tabular}

data sets but also degrades performance on seven. Principal components comes out the worst with improvement on five data sets and degradation on seven.

Table 3 ranks the attribute selection schemes. A pairwise comparison is made between each scheme and all of the others. The number of times each scheme is significantly more or less accurate than another is recorded and the schemes are ranked by the total number of "wins" minus "losses". From this table it can be seen that the Wrapper is clearly the best with 34 wins and only four losses against the other schemes. CFS and the consistency method are the only other schemes that have more wins than losses.

Table 4 shows the results for attribute selection with C4.5 and Table 5 shows the "wins" minus "losses" ranking for each scheme when compared against the others. The results are somewhat different than for naive Bayes. The best scheme for C4.5 is ReliefF which improves C4.5's performance on two data sets and degrades it on one. It is also top of the ranking with 22 wins and only seven losses against the other schemes. Consistency is the only other scheme that is ranked higher than using no feature selection with $\mathrm{C} 4.5$; it improves C4.5's performance on three data sets and degrades performance on three data 
Table 4: Results of attribute selection with $\mathrm{C} 4.5$

\begin{tabular}{|c|c|c|c|c|c|c|c|c|}
\hline Data Set & $\overline{\mathrm{C} 4.5}$ & $\mathrm{IG}$ & CFS & CNS & RLF & WRP & & $\mathrm{PC}$ \\
\hline 200 & 92.26 & 91.65 & 91.06 & 93.650 & 92.95 & 90.45 & - & 91.49 \\
\hline heart-stat & 78.67 & $84.52 \circ$ & $85.33 \circ$ & 84.110 & 820 & 82.11 & 0 & $82.22 \circ$ \\
\hline ionosphere & 89.74 & 89.4 & $91.09 \circ$ & 91.05 & 91.43 & 91.8 & $\circ$ & 88.8 \\
\hline diabetes & 73.74 & 73.92 & 73.67 & 73.71 & 73.58 & 73.5 & & 71.51 . \\
\hline vote & 96.46 & 95.84 & 95.65 & 95.98 . & $95.79 \bullet$ & 95.74 & - & 92.07. \\
\hline credit-g & 71.18 & 72.72 & $72.99 \circ$ & 72.2 & 71.63 & 72.23 & & 69.34 . \\
\hline soybean & 92.48 & 92.4 & 91.14 . & 92.43 & 92.43 & 92.19 & & $83.75 \bullet$ \\
\hline heart-c & 76.64 & $78.95 \circ$ & $79.11 \circ$ & $80.23 \mathrm{c}$ & $80.4 \circ$ & 77 & & 82.650 \\
\hline glass2 & 77.97 & 78.35 & 78.53 & 77.05 & 79.53 & 76.53 & & 66.41 • \\
\hline labor & 80.2 & 80.6 & 81 & 79.73 & 79.53 & 78.33 & & 88.6 \\
\hline lymph & 75.5 & $73.09 \bullet$ & 73.41 & 75.43 & 76.83 & 76.63 & & 74.6 \\
\hline breast-c & 73.87 & 73.75 & 73.7 & $72.24 \bullet$ & 72.77 & 73.43 & & 70.62 • \\
\hline segment & 96.9 & 96.81 & 96.94 & 96.87 & 96.89 & 96.92 & & 93.95 . \\
\hline anneal & 98.58 & 98.72 & 98.47 & 98.65 & 98.73 & 98.66 & & $96.26 \bullet$ \\
\hline horse colic & 85.44 & $84.18 \bullet$ & $83.94 \bullet$ & 84 & 84.9 & 84.14 & - & $78.18 \bullet$ \\
\hline
\end{tabular}

Table 5: Wins versus losses for accuracy of attribute selection with C4.5

\begin{tabular}{rlll}
\hline Scheme & $\begin{array}{l}\text { Wins- } \\
\text { Losses }\end{array}$ & Wins & Losses \\
\hline RLF & 15 & 22 & 7 \\
CNS & 12 & 20 & 8 \\
C4.5 & 7 & 23 & 16 \\
CFS & 5 & 21 & 16 \\
WRP & 5 & 17 & 12 \\
IG & 2 & 15 & 13 \\
PC & -46 & 12 & 58 \\
\hline
\end{tabular}

sets. CFS and the Wrapper are tied at fourth in the ranking. CFS improves C4.5's performance on four data sets (more than any other scheme) but also degrades performance on four datasets. The Wrapper improves performance on two datasets and degrades performance on three.

The success of ReliefF and consistency with $\mathrm{C} 4.5$ could be attributable to their ability to identify attribute interactions (dependencies). Including strongly interacting attributes in a reduced subset increases the likelihood that C4.5 will discover and use interactions early on in tree construction before the data becomes too fragmented. Naive Bayes, on the other hand, is unable to make use of interacting attributes because of its attribute independence assumption. Two reasons could account for the poorer performance of the Wrapper with C4.5. First, the nature of the search (forward selection) used to generate the ranking can fail to identify strong attribute interactions early on, with the result that the attributes involved are not ranked as highly as they perhaps should be. The second reason has to do with the Wrapper's attribute evaluation-five fold cross validation on the training data. Using cross validation entails setting aside some training data for evaluation with the result that less data is available for 
building a model.

Table 6: Size of trees produced by $\mathrm{C} 4.5$ with and without attribute selection.

\begin{tabular}{|c|c|c|c|c|c|c|c|c|}
\hline Data Set & C4.5 & $I G$ & CFS & CNS & RLF & WRP & & $\mathrm{PC}$ \\
\hline 200 & 15.64 & $13.22 \circ$ & 13.740 & $13.44 \circ$ & 13.040 & 13.98 & 0 & 13.020 \\
\hline heart-stat & 34.84 & $12.12 \circ$ & 11.980 & $13.52 \circ$ & $13.66 \circ$ & $\mathrm{I} 4.92$ & 0 & $4.82 \quad$ \\
\hline ionosphere & 26.58 & 21.840 & $16.64 \circ$ & 17.140 & 17.220 & 13.9 & 0 & 20.040 \\
\hline diabetes & 41.54 & $14.62 \circ$ & $15.92 \circ$ & $16.54 \circ$ & $16.74 \circ$ & 17.06 & 0 & $30.52 \mathrm{c}$ \\
\hline vote & 10.64 & $9.44 \quad 0$ & $8.64 \circ$ & 9.920 & 90 & 9.72 & 0 & 20.44 \\
\hline credit-g & 125.05 & 57.340 & $60.39 \circ$ & $61.82 \circ$ & $68.52 \circ$ & 63.48 & 0 & $10.98 \mathrm{c}$ \\
\hline soybean & 92.27 & 86.50 & 88.290 & 92.25 & 91.21 & 90.75 & & 88.84 \\
\hline heart-c & 42.34 & 19.720 & $19.45 \circ$ & 22.480 & $23.17 \circ$ & 24.2 & 0 & $8.16 \quad 0$ \\
\hline glass2 & 23.78 & $14.88 \circ$ & $16.28 \circ$ & $16.26 \circ$ & $17.12 \circ$ & 16.22 & 0 & 11.180 \\
\hline labor & 6.96 & $6.22 \quad 0$ & 6.10 & $6.18 \quad$ & $5.48 \quad \circ$ & 6.13 & 0 & $5.88 \quad$ \\
\hline lymph & 27.41 & 14.710 & $14.35 \circ$ & $12.26 \circ$ & $14.56 \circ$ & 14.43 & 0 & $18.18 \circ$ \\
\hline breast-c & 12.38 & 10.47 & 10.26 & 15.09 & 11.8 & 8.42 & 0 & 7.72 \\
\hline segment & 81.86 & 80.82 & 80.26 & 79.440 & 80.96 & 79.5 & & 119 \\
\hline anneal & 49.75 & 48.45 & 50.06 & $46.83 \circ$ & $46.73 \circ$ & 48.63 & & 38.94 \\
\hline horse colic & 8.57 & $21.18 \bullet$ & $25.75 \bullet$ & 8.81 & 20.64 & 20.9 & - & 6.42 \\
\hline
\end{tabular}

Table 7: Wins versus losses for C4.5 tree size

\begin{tabular}{rlll}
\hline Scheme & $\begin{array}{l}\text { Wins- } \\
\text { Losses }\end{array}$ & Wins & Losses \\
\hline PC & 21 & 47 & 26 \\
CFS & 15 & 30 & 15 \\
IG & 13 & 29 & 16 \\
RLF & 7 & 25 & 18 \\
CNS & 6 & 26 & 20 \\
WRP & 0 & 22 & 22 \\
C4.5 & -62 & 6 & 68 \\
\hline
\end{tabular}

Table 6 compares the size (number of nodes) of the trees produced by each attribute selection scheme against the size of the trees produced by C4.5 with no attribute selection. Smaller trees are preferred as they are easier to interpret. From Table 6 and the ranking given in Table 7 it can be seen that principal components produces the smallest trees, but since accuracy is generally degraded it is clear that models using the transformed attributes do not necessarily fit the data well. CFS is second in the ranking and produces smaller trees than C4.5 on 11 data sets with a larger tree on one dataset. Information gain, ReliefF and the Wrapper also produce smaller trees than $\mathrm{C} 4.5$ on 11 data sets but by and large produce larger trees than CFS. Consistency produces smaller trees than C4.5 on 12 data sets and never produces a larger tree. It appears quite low on the ranking because it generally produces slightly larger trees than the other schemes.

Table 8 shows the average number of attributes selected by each scheme for naive Bayes and Table 9 shows the "wins" versus "losses" ranking. Table 8 shows 
Table 8: Number of features selected for naive Bayes. Figures in brackets show the percentage of the original features retained.

\begin{tabular}{|c|c|c|c|c|c|c|c|c|}
\hline Data Sot & Orig & IG & RLF & CNS & $\mathrm{PC}$ & $\overline{C F S}$ & WR & \\
\hline 200 & 17 & $12.8(75 \%)$ & $12.5(74 \%)$ & $16.3(96 \%)$ & $4.7(28 \%)$ & $13.6(80 \%)$ & 10.5 & $(62 \%)$ \\
\hline heart-c & 13 & $7.1(55 \%)$ & $8.6(66 \%)$ & $8.7(67 \%)$ & $3.6(28 \%)$ & $7.2(55 \%)$ & 8.7 & $(67 \%)$ \\
\hline ionosphere & 34 & $7.9(23 \%)$ & $8.1 \quad(24 \%)$ & $10.5(31 \%)$ & $18.1(53 \%)$ & $12.6(37 \%)$ & 11.7 & $(34 \%)$ \\
\hline soybean & 35 & $30.9(88 \%)$ & $31.3(89 \%)$ & $32.7(93 \%)$ & $36 \quad(103 \%)$ & $25.8(74 \%)$ & 20.8 & $(59 \%)$ \\
\hline glass2 & 9 & $2.7(30 \%)$ & $3.2(35 \%)$ & $3.9(44 \%)$ & $4.5(50 \%)$ & $2.1(24 \%)$ & 1.9 & $(22 \%)$ \\
\hline vote & 16 & $1 \quad(6 \%)$ & $(11 \%)$ & $(16 \%)$ & $14.9(93 \%)$ & $1 \quad(6 \%)$ & 3 & $(19 \%)$ \\
\hline heart-stat & 13 & $7.8(60 \%)$ & $(71 \%)$ & $10.2(79 \%)$ & $2.6(20 \%)$ & (61\%) & 10 & $(77 \%)$ \\
\hline lymph & 18 & $16.6(92 \%)$ & $13.1(73 \%)$ & $14.3(79 \%)$ & $15.3(85 \%)$ & $(84 \%)$ & 13.1 & $(73 \%)$ \\
\hline - labor & 16 & $12.1(75 \%)$ & $13.6(85 \%)$ & $13.7(86 \%)$ & $4.3(27 \%)$ & $11.8(74 \%)$ & 9 & $(56 \%)$ \\
\hline diabctes & 8 & $2.7(34 \%)$ & $3.6(45 \%)$ & $4 \quad(50 \%)$ & $5.9(74 \%)$ & $2.8(35 \%)$ & 4.1 & $(53 \%)$ \\
\hline breast-c & 9 & $3.8(42 \%)$ & $7.4 \quad(82 \%)$ & $(63 \%)$ & $5.2(57 \%)$ & $2.7 \quad(30 \%)$ & 3.2 & $(36 \%)$ \\
\hline credit-g & 20 & $13.2(66 \%)$ & $14.3(72 \%)$ & $13.6(68 \%)$ & $19.9(100 \%)$ & $12.4(62 \%)$ & 10.7 & $(53 \%)$ \\
\hline segment & 19 & $11 \quad(58 \%)$ & $11.1(58 \%)$ & $5 \quad(26 \%)$ & $15.2(80 \%)$ & $7.9(42 \%)$ & 9.2 & $(48 \%)$ \\
\hline horse colic & 22 & $5.8(26 \%)$ & $4.1 \quad(18 \%)$ & $(18 \%)$ & $22.8(104 \%)$ & $(26 \%)$ & 6.2 & $(28 \%)$ \\
\hline anncal & 38 & $10.1(27 \%)$ & $(10 \%)$ & $(14 \%)$ & $38.9(103 \%)$ & $(19 \%)$ & 25.4 & $(67 \%)$ \\
\hline
\end{tabular}

Table 9: Wins versus losses for number of features selected for naive Bayes.

\begin{tabular}{rlll}
\hline Scheme & $\begin{array}{l}\text { Wins- } \\
\text { Losses }\end{array}$ & Wins & Losses \\
\hline CFS & 24 & 42 & 18 \\
IG & 11 & 35 & 24 \\
WRP & 9 & 35 & 26 \\
RLF & -1 & 30 & 31 \\
CNS & -15 & 21 & 36 \\
PC & -28 & 21 & 49 \\
\hline
\end{tabular}

that most schemes (with the exception of principal components) reduce the number of features by about $50 \%$ on average. Principal components sometimes increases the number of features (an artifact of the conversion of multi-valued discrete attributes to binary attributes). From Table 9 it can be seen that CFS chooses fewer features compared to the other schemes-retaining around $48 \%$ of the attributes on average. The Wrapper, which was the clear winner on accuracy, is third in the ranking-retaining just over $50 \%$ of the attributes on average

Table 10 shows the average number of features selected by each scheme for C4.5 and Table 11 shows the "wins" versus "losses" ranking. As to be expected, fewer features are retained by the schemes for C4.5 than for naive Bayes. CFS and the Wrapper retain about $42 \%$ of the features on average. ReliefF, which was the winner on accuracy, retains $52 \%$ of the features on average. As was the case with naive Bayes, CFS chooses fewer features for C4.5 than the other schemes (Table 11). ReliefF is at the bottom of the ranking in Table 11 but its larger feature set sizes are justified by higher accuracy than the other schemes.

It is interesting to compare the speed of the attribute selection techniques. We measured the time taken (in milliseconds ${ }^{3}$ ) to select the final subset of

${ }^{3}$ This is an approximate measure. Obtaining true cpu time from within a Java program is quite difficult. 
Table 10: Number of features selected for C4.5. Figures in brackets show the percentage of the original features retained.

\begin{tabular}{|c|c|c|c|c|c|c|c|c|c|c|}
\hline Data Set & Orig & $\mathrm{IG}$ & \multicolumn{2}{|c|}{ CFS } & \multicolumn{2}{|c|}{ CNS } & RLF & \multicolumn{2}{|c|}{ WRP } & $\mathrm{PC}$ \\
\hline 200 & 17 & $11.4(67 \%)$ & 9 & $(53 \%)$ & 11.2 & $(66 \%)$ & $10.5(62 \%)$ & 7.1 & $(42 \%)$ & $10.5(62 \%)$ \\
\hline heart-stat & 13 & $3.2(25 \%)$ & 3 & $(23 \%)$ & 3.6 & $(28 \%)$ & $5.6 \quad(43 \%)$ & 4.6 & $(35 \%)$ & $2.1(16 \%)$ \\
\hline ionosphere & 34 & $12.2(36 \%)$ & 6.9 & $(20 \%)$ & 9.3 & $(27 \%)$ & $8.7 \quad(26 \%)$ & 7.2 & $(21 \%)$ & $10.2(30 \%)$ \\
\hline abetes & 8 & $3.2(40 \%)$ & 3.4 & $(43 \%)$ & 3.6 & $(45 \%)$ & 3.9 & 3.8 & $(47 \%)$ & $5.9(74 \%)$ \\
\hline vote & 16 & $11.6(72 \%)$ & 9.6 & $(60 \%)$ & 6.5 & $\left(40^{\circ}\right.$ & $10.6(66 \%)$ & 8.6 & $(54 \%)$ & $11.2(70 \%)$ \\
\hline credit-g & 20 & $7.8(39 \%)$ & 6.7 & $(34 \%)$ & 8.1 & $(41 \%)$ & $9.1 \quad(45 \%)$ & 7.7 & $(39 \%)$ & $3.9(19 \%)$ \\
\hline soyboan & 35 & $29.5(84 \%)$ & 23.7 & $7(68 \%)$ & 35 & $(100 \%)$ & $32.4(93 \%)$ & 19.2 & $(55 \%)$ & $30.2(86 \%)$ \\
\hline & 13 & $3.9(30 \%)$ & 3.5 & $(27 \%)$ & 4 & $(31 \%)$ & $5.1 \quad(39 \%)$ & 5.9 & $(45 \%)$ & $3.8(29 \%)$ \\
\hline glass2 & 9 & $4.2(47 \%)$ & 4.6 & $(51 \%)$ & 4.4 & $(48 \%)$ & 4.7 & 4 & $(44 \%)$ & $4.2(47 \%)$ \\
\hline bor & 16 & $3.9(24 \%)$ & 2.8 & $(18 \%)$ & 6.6 & $(41 \%)$ & 6.5 & 3.3 & $(21 \%)$ & $3.5(22 \%)$ \\
\hline lymph & 18 & $6.8(38 \%)$ & 5.3 & $(30 \%)$ & 4 & $(22 \%)$ & $(25 \%)$ & 5.9 & $(33 \%)$ & $9.2(51 \%)$ \\
\hline breast-c & 9 & $4.4(49 \%)$ & 4 & $(44 \%)$ & 6.6 & $(73 \%)$ & $6.9 \quad(77 \%)$ & 3.98 & $(44 \%)$ & $4.4(49 \%)$ \\
\hline segment & 19 & $16.4(86 \%)$ & 11.9 & $9(63 \%)$ & 9.5 & $(50 \%)$ & $12.6(66 \%)$ & 9.2 & $(48 \%)$ & $16.4(86 \%)$ \\
\hline anneal & 38 & $16.6(44 \%)$ & 21.3 & $3(56 \%)$ & 15.5 & $(41 \%)$ & $20.4(54 \%)$ & 18.2 & $(48 \%)$ & $36.4(96 \%)$ \\
\hline
\end{tabular}

Table 11: Wins versus losses for number of features selected for C4.5

\begin{tabular}{rlll}
\hline Scheme & $\begin{array}{l}\text { Wins- } \\
\text { Losses }\end{array}$ & Wins & Losses \\
\hline CFS & 24 & 35 & 11 \\
WRP & 13 & 30 & 17 \\
CNS & 2 & 26 & 24 \\
IG & -8 & 18 & 26 \\
PC & -8 & 17 & 25 \\
RLF & -23 & 11 & 34 \\
\hline
\end{tabular}

attributes. This includes the time taken to generate the ranking and the time taken to cross validate the ranking to determine the best set of features. Table 12 shows the "wins" versus "losses" ranking for the time taken to select attributes for naive Bayes. CFS and information gain are much faster than the other schemes. As expected, the Wrapper is by far the slowest scheme. Principal components is also slow, probably due to extra data set pre-processing and the fact that initial dimensionality increases when multi-valued discrete attributes are present.

Table 12: Wins versus losses for time taken to select attributes for naive Bayes.

\begin{tabular}{rlll}
\hline Scheme & $\begin{array}{l}\text { Wins- } \\
\text { Losses }\end{array}$ & Wins & Losses \\
\hline CFS & 50 & 57 & 7 \\
IG & 49 & 56 & 7 \\
CNS & 13 & 38 & 25 \\
RLF & -10 & 29 & 39 \\
PC & -36 & 17 & 53 \\
WRP & -66 & 4 & 70 \\
\hline
\end{tabular}

Table 13 ranks the schemes by the time taken to select attributes for C4.5. It is interesting to note that the consistency method is the fastest in this case. 
Table 13: Wins versus losses for time taken to select attributes for C4.5

\begin{tabular}{rlll}
\hline Scheme & $\begin{array}{l}\text { Wins- } \\
\text { Losses }\end{array}$ & Wins & Losses \\
\hline CNS & 34 & 46 & 12 \\
IG & 29 & 42 & 13 \\
CFS & 25 & 40 & 15 \\
RLF & 12 & 36 & 24 \\
PC & -34 & 20 & 54 \\
WRP & -66 & 4 & 70 \\
\hline
\end{tabular}

While consistency does not rank attributes as fast as information gain, speed gains are made as a by-product of the quality of the ranking produced-with C4.5 it is faster to cross validate a good ranking than a poor one. This is because smaller trees are produced and less pruning performed early on in the ranking where the best attributes are. If poorer attributes are ranked near the top then C4.5 may have to "work harder" to produce a tree. This effect is not present with naive Bayes as model induction speed is not affected by attribute quality. Although ReliefF produces the best attribute rankings for $\mathrm{C} 4.5$, it is not as fast as information gain. The instance-based nature of the algorithm makes it slower at producing an attribute ranking.

\section{Results on Large Data Sets}

Figure 4 shows the results on the three large data sets for attribute selection with naive Bayes. Error bars denote the boundaries of a $95 \%$ confidence interval.

On the arrhythmia data set information gain, ReliefF, CFS and the Wrapper improve the performance of naive Bayes. Consistency gives roughly the same performance as naive Bayes and principal components degrades the performance of naive Bayes. From Table 14 it can be seen that only a small percentage of the original number of attributes is needed by naive Bayes on this data set-CFS retains just $3 \%$ of the features, and at the other end of the scale, information gain retains $22 \%$ of the features.

On the anonymous and internet-ads data sets the confidence intervals are much tighter due to larger test set sizes. There is no result for the Wrapper on the internet-ads data set because of the length of time it would take to run ${ }^{4}$. All methods, with the exception of principal components, perform at roughly the same level on these two data sets. CFS is fractionally better that the others on the internet-ads data set. On the anonymous data set the Wrapper and CFS are marginally better than the others. With the exception of principal components, Table 14 shows that CFS selects the smallest attribute sets for naive Bayes. CFS's bias in favour of predictive uncorrelated attributes is particularly well suited to naive Bayes.

\footnotetext{
${ }^{4}$ We estimated that about 140 days of runtime on our $1400 \mathrm{MHz}$ processor would be required in order to produce the attribute ranking
} 


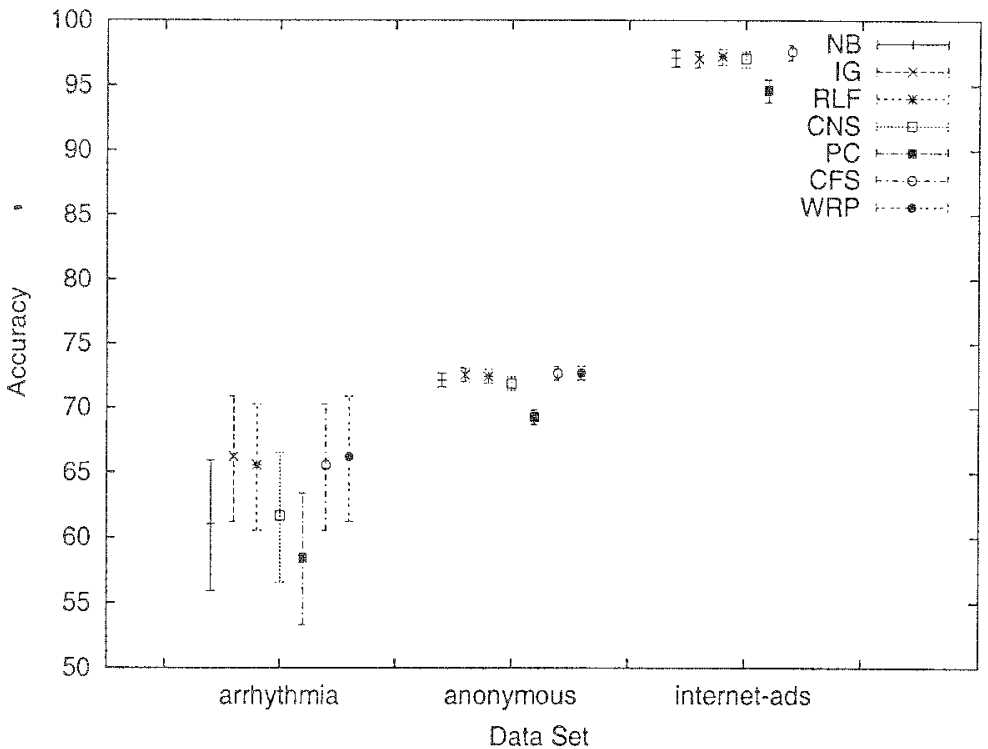

Figure 4: Feature selection results for naive Bayes on large data sets.

Table 14: Number of features selected for naive Bayes on large data sets. Figures in brackets show the percentage of the original features retained.

\begin{tabular}{|c|c|c|c|c|c|c|c|c|c|c|}
\hline Data Set & Orig & IG & $\mathrm{RLF}$ & $\mathrm{CN}$ & & $\mathrm{PC}$ & $\overline{C F}$ & & $\overline{W R}$ & \\
\hline arrhyth & 227 & $51(22 \%)$ & $13(6 \%)$ & 7 & $(3 \%)$ & $5(2 \%)$ & 6 & $(3 \%)$ & 47 & $(\%)$ \\
\hline anonyr & 293 & $12(4 \%)$ & $(6 \%)$ & 22 & $(8 \%)$ & $122(42 \%)$ & 11 & $(4 \%)$ & 182 & $(62 \%)$ \\
\hline
\end{tabular}




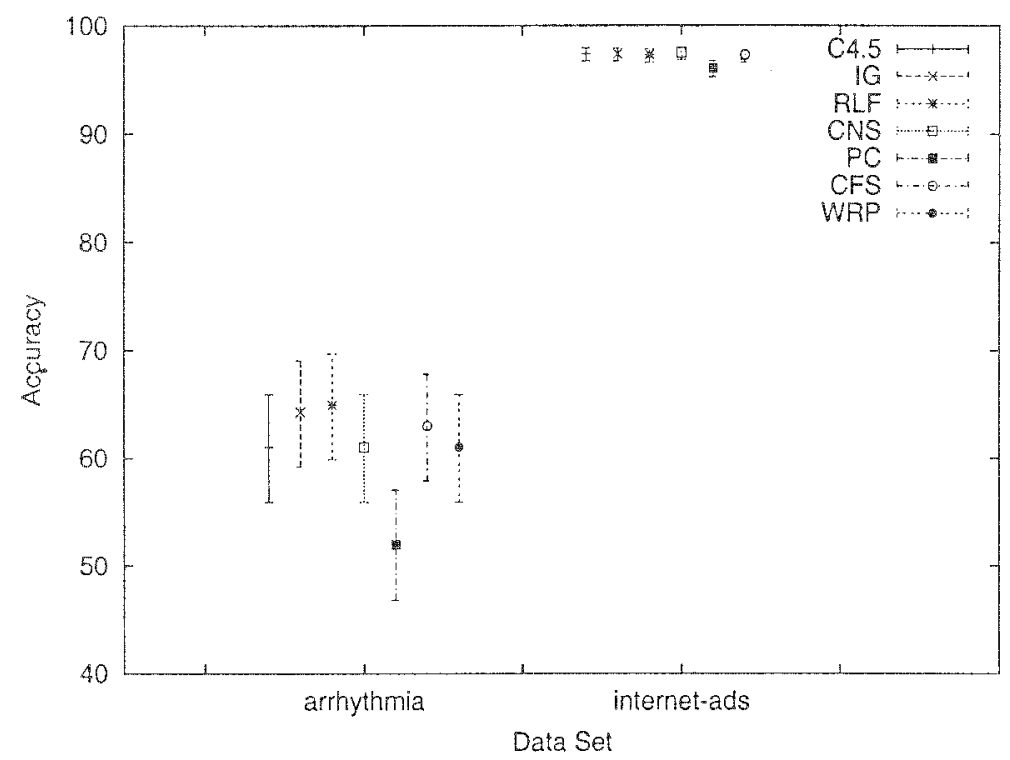

Figure 5: Feature selection results for $\mathrm{C} 4.5$ on large data sets.

Figure 5 shows the results on two of the three large data sets for attribute selection with C4.5. There are no results on the anonymous data set because the length of time needed to cross validate attribute rankings using $\mathrm{C} 4.5$ was prohibitive $^{5}$. On the arrhythmia data set information gain, ReliefF and CFS improve the performance of $\mathrm{C} 4.5$. ReliefF gives has the best performance on this data set. Table 15 shows the number of features retained by the attribute selection methods. As was the case for naive Bayes, good performance can be obtained on the arrhythmia data set given a small number of the original features. All methods, with the exception of principal components, perform equally well on the internet-ads data set. Figures in Table 15 and Table 16 help differentiate the methods. Disregarding principal components, the smallest feature sets are given by CFS and ReliefF. These two methods retain $5 \%$ and $43 \%$ of the original attributes respectively. RelefF, consistency and CFS make a small reduction in the size of C4.5's trees.

\section{Conclusions}

This paper has presented a benchmark comparison of six attribute selection techniques that produce ranked lists of attributes. The benchmark shows that

\footnotetext{
${ }^{5} \mathrm{C} 4.5$ 's runtime is dominated by the number of instances (roughly log-linear). A single ten fold cross-validation on the training data takes over an hour to complete and we estimated that over 40 days of processing would be required to evaluate a ranked list of attributes
} 
Table 15: Number of features selected for $\mathrm{C} 4.5$ on large data sets. Figures in brackets show the percentage of the original features retained.

\begin{tabular}{|c|c|c|c|c|c|c|c|c|c|}
\hline Data Set & Orig & IG & RL & & $\mathrm{CNS}$ & $\overline{\mathrm{PC}}$ & $\mathrm{CF}$ & & WRP \\
\hline arrhythmia & 227 & $28 \quad(12 \%)$ & 35 & $(15 \%)$ & $19 \quad(8 \%)$ & $2(1 \%)$ & 11 & $(5 \%)$ & $124 \quad(55 \%)$ \\
\hline internet-ads & 1557 & $1387(89 \%)$ & 663 & $(43 \%)$ & $1094(70 \%)$ & $16(1 \%)$ & 83 & $(5 \%)$ & - \\
\hline
\end{tabular}

Table 16: Size of trees produced by $\mathrm{C} 4.5$ with and without attribute selection on large data sets.

\begin{tabular}{lccccccc}
\hline Data Set & C4.5 & IG & CFS & CNS & RLF & WRP & PC \\
\hline arrhythmia & 67 & 61 & 63 & 63 & 53 & 61 & 33 \\
internet-ads & 47 & 47 & 43 & 43 & 43 & - & 49 \\
\hline
\end{tabular}

in general, attribute selection is beneficial for improving the performance of common learning algorithms. It also shows that, like learning algorithms, there is no single best approach for all situations. What is needed by the data miner is not only an understanding of how different attribute selection techniques work, but also the strengths and weaknesses of the target learning algorithm, along with background knowledge about the data (if available). All these factors should be considered when choosing an attribute selection technique for a particular application. For example, while the Wrapper using the forward selection search was well suited to naive Bayes, using a backward elimination search (which is better at identifying attribute interactions) would have been more suitable for C4.5.

Nevertheless, the results suggest some general recommendations. The wins versus losses tables show that, for accuracy, the Wrapper is the best attribute selection scheme, if speed is not an issue. Otherwise CFS, consistency and ReliefF are good overall performers. CFS chooses fewer features, is faster and produces smaller trees than the other two, but, if there are strong attribute interactions that the learning scheme can use then consistency or ReliefF is a better choice.

\section{References}

[1] H. Almuallim and T. G. Dietterich. Learning with many irrelevant features. In Proceedings of the Ninth National Conference on Artificial Intelligence, pages 547-552. AAAI Press, 1991.

[2] C. Blake, E. Keogh, and C. J. Merz. UCI Repository of Machine Learning Data Bases. University of California, Department of Information and Computer Science, Irvine, CA, 1998. [http://www. ics.uci.edu/ mlearn/MLRepository.html].

[3] Avrim Blum and Pat Langley. Selection of relevant features and examples in machine learning. Artificial Intelligence, 97(1-2):245-271, 1997. 
[4] M. Dash and H. Liu. Feature selection for classification. Intelligent Data Analysis, 1(3), 1997.

[5] S. Dumais, J. Platt, D. Heckerman, and M. Sahami. Inductive learning algorithms and representations for text categorization. In Proceedings of the International Conference on Information and Knowledge Management, pages $148-155,1998$.

[6] U. M. Fayyad and K. B. Irani. Multi-interval discretisation of continuousvalued attributes. In Proceedings of the Thirteenth International Joint Conference on Artificial Intelligence, pages 1022-1027. Morgan Kaufmann, 1993.

[7] M. A. Hall. Correlation-based feature selection for machine learning. PhD thesis, Department of Computer Science, University of Waikato, Hamilton, New Zealand, 1998.

[8] Mark Hall. Correlation-based feature selection for discrete and numeric class machine learning. In Proc. of the 17th International Conference on Machine Learning (ICML2000, 2000.

[9] K. Kira and L. Rendell. A practical approach to feature selection. In Proceedings of the Ninth International Conference on Machine Learning, pages 249-256. Morgan Kaufmann, 1992.

[10] Ron Kohavi and George H. John. Wrappers for feature subset selection. Artificial Intelligence, 97:273-324, 1997.

[11] I. Kononenko. Estimating attributes: Analysis and extensions of relief. In Proceedings of the Seventh European Conference on Machine Learning, pages 171-182. Springer-Verlag, 1994.

[12] P. Langley, W. Iba, and K. Thompson. An analysis of Bayesian classifiers. In Proc. of the Tenth National Conference on Artificial Intelligence, pages 223-228, San Jose, CA, 1992. AAAI Press. [Langley92.ps.gz, from http://www.isle.org/ langley/papers/bayes.aaai92.ps].

[13] H. Liu and R. Setiono. A probabilistic approach to feature selection: A filter solution. In Proceedings of the 13th International Conference on Machine Learning, pages 319-327. Morgan Kaufmann, 1996.

[14] J. R. Quinlan. C4.5: Programs for Machine Learning. Morgan Kaufmann, San Mateo, CA., 1993.

[15] M. Sikonja and I. Kononenko. An adaptation of relief for attribute estimation in regression. In Proceedings of the Fourteenth International Conference (ICML'97), pages 296-304. Morgan Kaufmann, 1997.

[16] Yiming Yang and Jan O. Pedersen. A comparative study on feature selection in text categorization. In International Conference on Machine Learning, pages 412-420, 1997. 